\title{
Competencias emocionales y rendimiento académico en los estudiantes de Educación Primaria
}

\author{
Antonio MARTíNEZ SÁNCHEZ
}

Universidad de Almería

(Recibido, el 1 de Febrero de 2018; Aceptado el 9 de Julio de 2018)

RESUMEN: El papel que juegan las emociones en el contexto educativo es fundamental, por lo que es necesario profundizar en la influencia de la inteligencia emocional a la hora de determinar el rendimiento académico de los estudiantes. El objetivo del presente estudio es analizar la relación de la inteligencia emocional sobre el rendimiento académico en una muestra de estudiantes de Educación Primaria. En concreto, tomaron parte en el estudio un total de 146 estudiantes del tercer ciclo de la etapa, con edades entre los 10 y 12 años, de tres centros públicos de Educación Primaria a los que se les aplicó el cuestionario TMMS-24. Los resultados encontrados muestran una asociación entre los niveles de inteligencia emocional y el rendimiento académico de los estudiantes, existiendo diferencias significativas entre hombres y mujeres a la hora de percibir las emociones. Un trabajo continuado por parte de los docentes de la capacidad de expresión, comprensión y regulación emocional puede mejorar el rendimiento académico del alumnado.

Palabras clave: Competencias emocionales, educación emocional, rendimiento académico, Educación Primaria.

\section{Emotional competences and academic performance in students of Primary Education}

\begin{abstract}
The role of emotions in the educational context is fundamental, so it is necessary to deepen the influence of emotional intelligence in determining the academic performance of students. The aim of the present study is to analyze the relationship of emotional intelligence to academic achievement in a sample of Primary Education students. Specifically, a total of 146 students from the third cycle of the stage, aged between 10 and 12 years, from three public Primary Education centers took part in the study, to which the TMMS-24 questionnaire was applied. The results show an association between the levels of emotional intelligence and students' academic performance, with significant differences between men and women when perceiving emotions. Continued work by teachers on the ability of expression, understanding and emotional regulation can improve the academic performance of students.
\end{abstract}

Keywords: Emotional competencies, emotional education, academic achievement, Primary Education.

Correspondencia: Antoni Martínez Sánchez. Universidad de Almería. ams820@ual.es 


\section{Introducción}

En los últimos años se han producido cambios significativos en el proceso de enseñanzaaprendizaje para favorecer una formación integral de los estudiantes mediante el desarrollo de sus conocimientos, capacidades y habilidades desde los ámbitos cognitivo, social, físico, moral $\mathrm{y}$ afectivo. Se ha evolucionado de un modelo educativo centrado en la transmisión de conocimientos a otro basado en la adquisición y desarrollo de competencias. Este concepto de competencia implica la capacidad de adaptarse a una realidad en continuo cambio e integrarse con éxito en diferentes contextos, para lo que se requiere una gestión adecuada de las emociones. Por tanto, adquiere especial relevancia el desarrollo de competencias emocionales en las aulas de educación primaria.

Se entiende por competencias emocionales "el conjunto de conocimientos, capacidades, habilidades y actitudes necesarias para tomar conciencia, comprender, expresar y regular de forma apropiada los fenómenos sociales" (Bisquerra, 2009, p.146). Estas competencias se pueden agrupar en cinco dimensiones: conciencia emocional, regulación emocional, autonomía personal, inteligencia interpersonal y habilidades de vida y bienestar (Bisquerra y Pérez, 2007). El dominio de las competencias emocionales entre los estudiantes favorecen, entre otros aspectos, el proceso de aprendizaje, las relaciones interpersonales y la resolución de problemas (Bisquerra y Pérez, 2007; Fernández y Extremera, 2005).

Las competencias emocionales están basadas en el constructo inteligencia emocional (IE), añadiendo otros atributos emocionales y sociales (Bisquerra, Pérez y García, 2015). El término IE es un modelo teórico, propio del campo de estudio de la psicología, que se ha extendido en los últimos años en el ámbito educativo. Es concebida como una inteligencia basada en el uso adaptativo de las emociones de manera que ayuda al individuo en solución de problemas, y facilita la adaptación al medio que le rodea de una forma eficaz (Grewal y Salovey, 2005). En el estudio científico de la IE, se ha adoptado el modelo de habilidad, propuesto por Mayer y Salovey (Mayer, Caruso y Salovey, 1999), que conceptualiza el término como la capacidad para valorar y expresar las emociones, acceder y generar sentimientos que faciliten el pensamiento, entender la emoción y el crecimiento emocional, así como la capacidad para regular las emociones y promover el crecimiento emocional e intelectual (Mayer y Salovey, 1997). Las habilidades emocionales que se recogen en la IE, permiten un mejor procesamiento y asimilación de la información emocional en situaciones vitales de una persona como puede ser un mejor desempeño académico (Mayer, Roberts y Barsade, 2008; Salovey, Mayer y Caruso, 2002).

Para evaluar la IE se ha utilizado tradicionalmente medidas de autopercepción sobre su propia IE, es lo que se denomina Inteligencia Emocional Percibida -IEP- (Mayer, Salovey y Caruso, 2000; Salovey el al., 2002). El instrumento de elección empleado para medir la IEP es el Trait Meta-Mood Scale (TMMS) que se compone de tres subescalas: atención, claridad y reparación emocional, así como la versión reducida y traducida al castellano, el TMMS-24 (Fernández, Extremera y Ramos, 2004) que tienen por objetivo obtener una estimación personal sobre los aspectos reflexivos de la experiencia emocional de los individuos. Dentro de la conceptualización de la IEP, ésta es considerada como una habilidad que se incrementa con la experiencia y edad (Mayer y Salovey, 1997). 
En los últimos años, una de las líneas de investigación que mayor interés ha suscitado es el estudio del papel que juegan las emociones en el ámbito educativo y su posible predicción en el rendimiento académico. En este sentido, definimos como rendimiento académico (RA) al nivel de conocimientos y destrezas que poseen los estudiantes. Existe evidencia empírica del papel que juegan las emociones en el RA del alumnado, y como la IEP influye de forma directa e indirecta en diversas áreas de funcionamiento, contribuyendo al bienestar y la adaptación del estudiante al sistema educativo (Extremera y Fernández-Berrocal, 2004; Jiménez y López, 2009).

Tras una revisión bibliográfica encontramos, por una parte, diversos estudios que han mostrado que las competencias emocionales afectan a los estudiantes en el contexto escolar, concretamente la influencia que tiene sobre el éxito o fracaso escolar (Fernández, Extremera y Ramos, 2004; Gil, Palomera y Brackett, 2006; Pérez y Castejón, 2007; Petrides, Frederickson y Furnham, 2004). Por otra, otras investigaciones encuentran resultados dispares a la hora de relacionar ambas variables. Diversos autores establecen una correlación positiva entre IEP y RA (Durán, Extremera, Rey, Fernández y Montealban., 2006; Gil, Palomera y Brackett, 2006; Nasir y Masrur, 2010; Pérez y Castejón, 2006), mientras que otros estudios hallan una correlación negativa (Sánchez, Rodríguez y Padilla, 2007). Incluso podemos encontrar estudios en los que no se establece relación alguna entre estas variables (Ferragut y Fierro, 2012; Font, 2013).

En el caso de estudiantes adolescentes, también existen disparidad de resultados. Algunos trabajos encuentran una relación débil entre ambas variables (Buenrostro, Valadez, Soltero, Nava, Zambrano, y García, 2012; Jiménez y López, 2013). En este sentido, el estudio de Otero, Martín, León y Vicente (2009), realizado sobre una muestra de alumnado de $1^{\circ}$ y $2^{\circ}$ curso de Educación Secundaria Obligatoria, en el que encontraron bajas correlaciones entre la inteligencia emocional y medidas globales del rendimiento, y ninguna significativa entre ellas. Se utilizó como instrumento el TMMS-24 para evaluar la inteligencia emocional, y para el rendimiento académico la nota media y el número de suspensos del curso anterior. Al introducir la variable género para analizar la relación de la IEP entre los estudiantes adolescentes, los resultados demuestran que se evidencian diferencias en los valores de IEP (Salguero, Fernández-Berrocal, Balluerka y Aritzeta, 2010). Por otra parte, destacar los resultados y conclusiones de Ferrando, Prieto, Almeida, Ferrándiz, Bermejo, López, Hernández, Sainz y Fernández (2010) en el que hallaron una correlación positiva significativa entre el rasgo de inteligencia emocional y desempeño académico en estudiantes del último ciclo de la etapa de Educación Primaria.

A partir de estas aportaciones, consideramos necesario realizar el presente estudio para conocer las semejanzas o discrepancias en los resultados. El objeto es conocer la posible predicción que desempeñan las competencias emocionales en el rendimiento académico del alumnado de último ciclo de educación primaria. Concretamente los objetivos de investigación que se persiguen son:

Analizar el nivel de inteligencia emocional de los estudiantes del tercer ciclo de Primaria de una forma diferenciada en función del género.

Profundizar en la exploración respecto a la posible asociación entre IEP y RA, y comprobar si tales relaciones se replican en nuestra muestra de estudiantes, dada la escasez de estudios en este subgrupo de edad (10-12 años).

Por último, se analiza si la supuesta asociación entre ambas variables (IEP y RA) está mediada por la existencia de diferencias en función del género. 


\section{Método}

\section{Participantes}

Teniendo en cuenta diferentes zonas geográficas de la provincia de Almería, se obtiene la muestra compuesta por un total de 146 sujetos, alumnado de quinto $(54,8 \%)$ y sexto $(45,2 \%)$ curso, de tres centros públicos de Educación Primaria de la provincia de Almería, con edades comprendidas entre los 10 y los 12 años, seleccionados por accesibilidad. Como podemos observar en la Tabla 1, del total de la muestra, el 58,9\% fueron hombres y el 41,1\% mujeres. En cuanto al rendimiento académico se observó que el mayor porcentaje de sujetos $(56,2 \%)$ no suspendieron ninguna asignatura, mientras que entre los que suspendieron una o cuatro asignaturas se encontraban el $43,8 \%$ de la muestra.

Tabla 1. Estadísticos descriptivos de la muestra

\begin{tabular}{llcc}
\hline & & $\mathrm{N}$ & $\%$ \\
\hline Género & Hombre & 86 & 58,9 \\
& Mujer & 60 & 41,1 \\
\hline Curso & Quinto & 80 & 54,8 \\
& Sexto & 66 & 45,2 \\
\hline Asignaturas & Cero asignaturas & 82 & 56,2 \\
Suspensas & Una asignatura & 22 & 15 \\
& & 16 & 11,8 \\
& Tres asignaturas & & \\
& Cuatro asignaturas & & \\
\hline
\end{tabular}

\section{Diseño}

Para el diseño del estudio hemos optado por una metodología selectiva, siendo de tipo exploratorio mediante encuesta transversal, en la búsqueda de la relación que guardan las competencias emocionales y el rendimiento académico del alumnado de último ciclo de educación primaria.

\section{Instrumentos}

Para recoger la información más relevantes de las características de la muestra se utilizó un cuestionario sociodemográfico elaborado ad hoc que nos proporcionaba información sobre el género, curso y número de asignaturas suspensas.

El rendimiento académico se evaluó en función del número de asignaturas suspensas al finalizar el curso anterior, como predictor del rendimiento actual del alumnado (García, Alvarado y Jiménez, 2000), aunque el RA también depende de otras variables que están relacionadas con la motivación, la personalidad y la inteligencia emocional de los estudiantes (Otero et al., 2009).

Para evaluar las competencias emocionales se utilizó la Escala de Inteligencia Emocional TMMS-24 (Fernández-Berrocal, Extremera y Ramos, 2004). Consta de 24 ítems 
que se subdividen en tres dimensiones relacionadas con la inteligencia emocional:

1. Percepción emocional: constituye la habilidad para identificar y reconocer los sentimientos propios como ajenos.

2. Comprensión emocional: implica la habilidad para desplegar un repertorio de emociones, etiquetarlas y reconocer en qué categorías se agrupan los sentimientos.

3. Regulación emocional: capacidad de apertura hacia los sentimientos, tantos positivos como negativos, y reflexionar sobre éstos para aprovecharlos de manera positiva.

Las puntaciones de cada una de estas subescalas se clasifican en tres rangos (alto, medio y bajo) con diferentes puntos de corte tanto para hombres como para mujeres. Cuenta con una escala Likert de 1 (nada de acuerdo) a 5 (totalmente de acuerdo) puntos que representa el grado de acuerdo con cada una de las afirmaciones sobre los sentimientos y emociones.

\section{Procedimiento}

El procedimiento a seguir fue idéntico en los tres centros educativos. En primer lugar, una vez que seleccionamos los centros, obtuvimos el consentimiento informado de la dirección del colegio y de los padres, madres o tutores legales del alumnado, a los que hicimos participes de los objetivos y resultados del estudio. La implementación del cuestionario se llevó a cabo en dos sesiones de unos 60 minutos cada una. La primera sesión se realizó con el alumnado de quinto curso. En primer lugar les explicamos en qué consistía el cuestionario y la forma en la que tenían que cumplimentarlo y por último se fueron leyendo las preguntas una a una y aclarando dudas y poniendo ejemplos prácticos de cada situación. En la segunda sesión se encuestó al alumnado de sexto curso del mismo modo que en el caso anterior.

La valoración de los niveles de competencia en los estudiantes, a partir de los resultados obtenidos en los instrumentos, se realizó en base a la baremación establecida en el test estandarizado.

\section{Análisis de datos}

Para el análisis y tratamiento de los datos utilizamos el programa estadístico SPSS.23, los parámetros descriptivos fueron realizados mediante el análisis de frecuencias. Para el análisis de puntuaciones medias, así como para conocer la diferencia entre ellas, se aplicó la prueba $t$ de Student para muestras independientes, en función de la variable género. A continuación, se llevó a cabo un ANOVA para analizar la diferencia de puntuación media entre grupos, sobre cada una de las tres dimensiones que valora el cuestionario: Percepción, Comprensión y Regulación, complementada por el tamaño del efecto. Por último, para conocer las diferencias entre sexos en relación a la inteligencia emocional y rendimiento académico se realizó un análisis univariante de la varianza. 


\section{Resultados}

En primer lugar se muestran los resultados obtenidos con la muestra del estudio, diferenciada por género, respecto a las diferentes dimensiones inteligencia emocional mediante la escala de inteligencia emocional (TMMS-24). Tal y como se observa en la tabla 2, fueron las mujeres quienes obtuvieron un media superior respecto a los hombres en las tres dimensiones de la inteligencia emocional. Además, estas diferencias encontradas son estadísticamente significativas $(\mathrm{p}<, 05)$ tanto en las dimensiones de percepción, comprensión y regulación en función del sexo.

Tabla 2. Inteligencia emocional (TMMS- $\left.{ }_{24}\right)$ con el sexo. Estadistica descriptiva y Prueba $t$

\begin{tabular}{lcccccc}
\hline & Sexo & N & Media & DT & $t$ & $p$ \\
\hline \multirow{2}{*}{ Percepción } & Hombres & 86 & 29,00 & 6,25 & & \\
& Mujeres & 60 & 33,86 & 3,85 & & \\
& & & & & & \\
\multirow{2}{*}{ Comprensión } & Hombres & 86 & 39,04 & 6,67 & & \\
& Mujeres & 60 & 42,06 & 4,62 & $-3,230$ &, $002^{*}$ \\
& Hombres & 86 & 23,18 & 4,37 & & \\
\multirow{2}{*}{ Regulación } & Mujeres & 60 & 25,26 & 4,26 & & \\
& & & & & & \\
& & & & & & \\
\hline
\end{tabular}

$* \mathrm{p}<, 05$

En lo que se refiere al análisis de la relación entre inteligencia emocional y rendimiento académico, como se puede observar en la tabla 3, en la dimensión percepción, los que menos puntuaron son los sujetos que tienen cuatro asignaturas suspensas $(\mathrm{M}=27,84 ; \mathrm{DT}=4,62)$. En comprensión, al contrario que en la anterior, fueron los sujetos que no suspendieron ninguna asignatura los que menos puntuaron en esta dimensión, obteniendo una media de $(\mathrm{M}=39,21$; $\mathrm{DT}=6,23)$ puntos. Por último, en la dimensión de la regulación vemos que los sujetos que obtuvieron mayores puntuaciones medias, fueron los que suspendieron tres asignaturas $(\mathrm{M}=27,37 ; \mathrm{DT}=5,11)$. Tanto en las dimensiones de percepción $(\mathrm{p}=, 000)$, como en la de Regulación emocional $(\mathrm{p}=, 005)$ encontramos que existen diferencias estadísticamente significativas. 
Tabla 3. Inteligencia emocional (TMMS-24) con el número de asignaturas suspensas.

Estadística descriptiva ANOVA

\begin{tabular}{|c|c|c|c|c|c|c|}
\hline & Suspensos & $\mathrm{N}$ & $\mathrm{M}$ & DT & $F$ & $p$ \\
\hline \multirow{4}{*}{ Percepción } & Cero asignaturas & 82 & 30,53 & 6,47 & \multirow{4}{*}{7,402} & \multirow{4}{*}{, $000^{*}$} \\
\hline & Una asignatura & 22 & 33,45 & 1,87 & & \\
\hline & Tres asignaturas & 16 & 35,12 & 4,80 & & \\
\hline & Cuatro asignaturas & 26 & 27,84 & 4,62 & & \\
\hline \multirow{4}{*}{ Comprensión } & Cero asignaturas & 82 & 39,21 & 6,23 & \multirow{4}{*}{2,26} & \multirow{4}{*}{, 083} \\
\hline & Una asignatura & 22 & 41,36 & 4,28 & & \\
\hline & Tres asignaturas & 16 & 42,87 & 3,87 & & \\
\hline & Cuatro asignaturas & 26 & 41,15 & 7,38 & & \\
\hline \multirow{4}{*}{ Regulación } & Cero asignaturas & 82 & 23,92 & 4,43 & \multirow{4}{*}{4,512} & \multirow{4}{*}{, $005^{*}$} \\
\hline & Una asignatura & 22 & 22,27 & 2,79 & & \\
\hline & Tres asignaturas & 16 & 27,37 & 5,11 & & \\
\hline & Cuatro asignaturas & 26 & 23,84 & 4,28 & & \\
\hline
\end{tabular}

A continuación se analizan las diferencias entre sexos en relación a la inteligencia emocional y el rendimiento académico. A nivel descriptivo, como aparece reflejado en la Tabla 4, al rendimiento académico se le ha otorgado el valor 1 a los estudiantes que no han suspendido ninguna asignatura, el valor 2 a los que han suspendido una y el valor 3 a los que han suspendido más de una asignatura. En el análisis de las diferencias de medias por género, se observaron diferencias estadísticamente significativas en algunas de las variables evaluadas. En la puntuación de la comprensión, aparecieron diferencias estadísticamente significativas ( $p$ $=.003$ ) entre el grupo de mujeres. Asimismo, en relación a la regulación, se apreciaron diferencias estadísticamente significativas $(p=.002)$ en dicho grupo. 
Tabla 4. Diferencias entre sexos en relación a inteligencia emocional y rendimiento académico. Análisis univariante de la varianza

\begin{tabular}{|c|c|c|c|c|c|c|c|c|}
\hline & Sexo & Suspensos & $\mathrm{N}$ & M & DT & $F$ & $p$ & $\eta^{2}$ \\
\hline \multirow{6}{*}{ Percepción } & \multirow{3}{*}{ Hombres } & 1 & 6 & ,00 & 00 & \multirow{3}{*}{1,466} & \multirow{3}{*}{,237 } & \multirow{3}{*}{, 03} \\
\hline & & 2 & 66 & 1,09 & 1,68 & & & \\
\hline & & 3 & 14 &, 71 & 1,43 & & & \\
\hline & \multirow{3}{*}{ Mujeres } & 1 & 2 & 3,00 & ,00 & \multirow{3}{*}{1,546} & \multirow{3}{*}{,222 } & \multirow{3}{*}{, 051} \\
\hline & & 2 & 30 & 1,26 & 1,72 & & & \\
\hline & & 3 & 28 & 1,71 & 1,35 & & & \\
\hline \multirow{5}{*}{ Comprensión } & \multirow{3}{*}{ Hombres } & 1 & 4 & ,00 &, 00 & \multirow{3}{*}{,789 } & \multirow{3}{*}{,458 } & \multirow{3}{*}{, 019} \\
\hline & & 2 & 18 &, 88 & 1,71 & & & \\
\hline & & 3 & 64 & 1,03 & 1,62 & & & \\
\hline & \multirow[b]{2}{*}{ Mujeres } & 2 & 8 & 3,00 & 1,30 & \multirow[b]{2}{*}{9,357} & \multirow[b]{2}{*}{, $003 *$} & \multirow[b]{2}{*}{, 139} \\
\hline & & 3 & 52 & 1,30 & 1,47 & & & \\
\hline \multirow{5}{*}{ Regulación } & & 1 & 42 & 95 & 1,54 & \multirow{2}{*}{, 000} & \multirow{2}{*}{,995 } & \multirow{2}{*}{,000 } \\
\hline & Homores & 2 & 44 &, 95 & 1,68 & & & \\
\hline & \multirow{3}{*}{ Mujeres } & 1 & 20 & 2,20 & 1,70 & \multirow{3}{*}{6,861} & \multirow{3}{*}{, $002 *$} & \multirow{3}{*}{,194 } \\
\hline & & 2 & 36 & 1,00 & 1,30 & & & \\
\hline & & 3 & 4 & 3,00 &, 00 & & & \\
\hline
\end{tabular}

\section{Discusión y Conclusiones}

El propósito fundamental del estudio es analizar la relación existente entre las competencias emocionales y el rendimiento académico en los estudiantes del último ciclo de educación primaria.

En primer lugar, respecto al primer objetivo del estudio, analizar las competencias emocionales de los estudiantes, podemos afirmar que, en general, poseen un adecuado nivel de inteligencia emocional. En este sentido, destacar en ambos sexos la excelente comprensión emocional, mientras que, en el caso de los hombres, deben mejorar en la dimensión de regulación emocional. Los resultados obtenidos evidencian que las mujeres obtienen puntuaciones medias más altas que los hombres en los tres factores del TMMS-24 que miden la inteligencia emocional, en línea con el estudio presentado por Otero, Martín, León y Vicente (2009). En el caso de las dimensiones de comprensión y regulación emocional estas diferencias 
entre hombres y mujeres son estadísticamente significativas. Por tanto, estos resultados corroboran la existencia de diferencias en los valores de la inteligencia emocional percibida en función del sexo (Salguero et al., 2010; Extremera, Durán y Rey, 2007; Molero, Ortega y Moreno, 2010).

En relación al segundo de los objetivos del estudio, la exploración de la asociación entre inteligencia emocional percibida y rendimiento académico, los resultados determinan la existencia de relaciones moderadas entre ambas variables corroborando las conclusiones del estudio realizado por Ferrando et al. (2010) sobre este grupo de edad de estudiantes. En nuestro estudio, estas diferencias son significativas tanto en las dimensiones de percepción como de regulación emocional. En el caso de la percepción, estos resultados coinciden con el estudio de Sánchez, Rodríguez y Padilla (2007), que emplearon la escala TMMS-24 para medir la Inteligencia Emocional de estudiantes universitarios. Estas investigaciones hallaron una correlación negativa y significativa entre la dimensión percepción y el promedio de las calificaciones del curso anterior de cada estudiante. De hecho, si observamos los criterios de corrección del TMMS-24 respecto a la dimensión percepción, vemos que es la única variable en la que se especifica que una puntuación que supere la máxima del rango adecuado no resulta excelente, como en los otros casos, sino perjudicial, pues la persona en este caso presta demasiada atención.

En cuanto al tercer y último objetivo, analizar la asociación entre inteligencia emocional y rendimiento académico en función del sexo, los resultados muestran que, en el caso de las mujeres, tanto en las dimensiones de comprensión y regulación emocional existen diferencias estadísticamente significativas respecto a los hombres.

En definitiva, a través de nuestra investigación se hace patente la existencia de cierta asociación entre los niveles de inteligencia emocional y el rendimiento académico. Por ello, creemos firmemente que un trabajo continuado de la capacidad de expresión, comprensión y regulación emocional puede mejorar el rendimiento académico del alumnado, por lo que consideramos necesario que se incorpore a las prácticas docentes estas habilidades que permitan la obtención de mejores calificaciones.

\section{Limitaciones}

El presente estudio tiene algunas limitaciones. Además de la representatividad de la muestra, que sólo puede ser generalizable a tres centros de educación primaria de Almería, por lo que sería necesario replicar estos resultados en una muestra mayor y más heterogénea. Otra limitación es que únicamente se ha evaluado el rendimiento académico a través del número de asignaturas suspendidas durante el curso 2011/12, por lo que podría ampliarse, tanto a los cursos anteriores, así como al empleo de nota media de los estudiantes, la calificación en las diferentes materias que componen la educación primaria y/o la presencia de alumnado repetidor de curso para medir dicho rendimiento. 


\section{Referencias}

Bisquerra, R. (2009). Psicopedagogía de las emociones. Madrid: Síntesis.

Bisquerra, R., y Pérez-Escoda, N. (2007). Las competencias emocionales. Educación XXI, 10, 61-82.

Bisquerra, R., Pérez-González, J. C., y García-Navarro, E. (2015). Inteligencia emocional en educación. Madrid: Síntesis.

Buenrostro-Guerrero, A. E., Valadez-Sierra, M. D., Soltero-Avelar, R., Nava-Bustos, G., Zambrano-Guzmán, R., y García-García, A. (2012). Inteligencia emocional y rendimiento académico en adolescentes. Revista de Educación y Desarrollo, 20, 29-37.

Cazalla, L., y Molero, D. (2014). Inteligencia emocional percibida, ansiedad y afectos en estudiantes universitarios. Revista Española de Orientación y Psicopedagogía, 25(3), 56-73. doi:10.5944/reop.vol.25.num.3.2014.13858.

Durán, A., Extremera, N., Rey, L., Fernández-Berrocal, P., y Montealban, F.M. (2006). Predicting academic burnout and engagement in educational settings: Assessing the incremental validity of perceived emotional intelligence beyond perceived.

Extremera, N., Durán, A., y Rey, L. (2007). Inteligencia emocional y su relación con los niveles de burnout, engagement y estrés en estudiantes universitarios. Revista de Educación, 342, 239-256.

Extremera, N., Durán, A., y Rey, L. (2009). The moderating effect of trait meta-mood and perceived stress on life satisfaction. Personality and Individual Differences, 47(2), 116121. doi: 10.1016/j.paid.2009.02.007

Extremera, N., y Fernández-Berrocal, P. (2004). El papel de la inteligencia emocional en el alumnado. Revista Electrónica de Investigación Educativa, 6(2), 0-17.

Fernández-Berrocal, P., y Extremera. N. (2005). La Inteligencia Emocional y la Educación de las emociones desde el modelo de Mayer y Salovey. Revista Interuniversitaria de Formación Del Profesorado, 19 (3), 63-93.

Fernández-Berrocal, P., Extremera, N., y Ramos, N. (2004). Validity and reliability of the Spanish modified versión of the Trait Meta-Mood Scale. Psychological Reports, 94(3), 751-755.

Ferrando, M., Prieto, M., Almeida, L., Ferrándiz, C., Bermejo, R., López-Pina, J., Hernández, D., Sainz, M., Fernández, M. (2010). Trait Emotional Intelligence and Academic Performance: Controlling for the effects of IQ, personality, and self-concept. Journal of Psychoeducational Assessment. July 29. doi: 10.1177/0734282910374707

Ferragut, M., y Fierro, A. (2012). Inteligencia emocional, bienestar personal y rendimiento académico en preadolescentes. Revista Latinoamericana de Psicología, 44(3), 95-104.

Font, T. (2013). Intelligència emocional, engagement i èxit acadèmic. Revista de Psicologia, Ciències de l'Educació $i$ de l'Esport Blanquerna, 31(2), 59-64.

García M.V., Alvarado J.M. y Jiménez A. (2000). La predicción del rendimiento académico: regresión lineal versus regresión logística. Psicothema, 12, 248-252.

Gil-Olarte, P., Palomera, R. y Brackett, M. (2006). Relating emotional intelligence to social competence and academic achievement in high school students. Psichothema, 18, supl., 118-123. 
Greway, D. y Salovey, P. (2005). "Feeling Smart: The Science of Emotional Intelligence". American Scientist, 93, 330-339.

Jiménez-Morales, I., y López-Zafra, E. (2009). Inteligencia emocional y rendimiento escolar: estado actual de la cuestión. Revista Latinoamericana de Psicología, 41(1), 69-80. doi: 10.14349/rlp.v41i1.556

Mayer, J. D., Caruso, D. R., y Salovey, P. (1999). Emotional intelligence meets traditional standards for an intelligence. Intelligence, 27(4), 267-298. doi: 10.1016/S01602896(99)00016-1.

Mayer, J. D., Roberts, R. D. y Barsade, S. G. (2008). Human abilities: Emotional intelligence. Annual Review of Psychology, 59, 507-536. doi:10.1146/annurev.psych.59.103006.093646

Mayer, J. D., y Salovey, P. (1997). What is emotional intelligence? En P. Salovey y D. Sluyter (Eds.), Emotional development and emotional intelligence: Educational applications (pp. 3-31). New York: Basic Books.

Mayer, J. D., Salovey, P., y Caruso, D. R. (2000). Models of emotional intelligence. En R. J. Sternberg (Ed.), Handbook of intelligence (pp. 396-420). Cambridge: Cambridge University Press.

Molero, D., Ortega-Álvarez, F., y Moreno-Romero, M. R. (2010). Diferencias en la adquisición de competencias emocionales en función del género. Revista Electrónica de Investigación y Docencia (REID), 3, 165-172.

Nasir, M. y Masrur, R. (2010). An exploration of emotional intelligence of the students of IIUI in relation to gender, age and academic achievement. Bulletin of education and research, 32(1), 37-51.

Otero, C, Martín, E., León, B. y Vicente, F. (2009). Inteligencia emocional y rendimiento académico en estudiantes de enseñanza secundaria. Diferencias de género. Revista Galego-Portuguesa de Psicoloxía e Educación, 17, 275-284.

Pérez-Pérez, N., y Castejón, J. L. (2006a). La inteligencia emocional como predictor del rendimiento académico en estudiantes universitarios. Ansiedad y Estrés, 12(2-3), 393400. doi: 1320858454_847

Petrides, K. V., Frederickson, N. y Furnham, A. (2004). The role of trait emotional intelligence in academic performance and deviant behavior at school. Personality and Individual Differences, 36 (2), 277-293.

Salovey, P., Mayer, J. D., y Caruso, D. R. (2002). The positive psychology of emotional intelligence. En C. R. Snyder y S. J. Lopez (Eds.), The handbook of positive psychology (pp. 159-171). New York: Oxford University Press. doi: 10.1093/ oxfordhb/9780195187243.013.0022

Salovey, P., Stroud, L. R., Woolery, A., y Epel, E. S. (2002). Perceived emotional intelligence, stress reactivity, and symptom reports: Further explorations using the trait meta-mood scale. Psychology \& Health, 17(5), 611-627. doi: 10.1080/08870440290025812

Salguero, J. M., Fernández-Berrocal, P., Balluerka, N., y Aritzeta, A. (2010). Measuring perceived emotional intelligence in the adolescent population: Psychometric properties of the trait meta-mood scale. Social Behavior and Personality, 38(9), 1197-1209. doi: 10.2224/sbp.2010.38.9.1197

Sánchez, M., Rodríguez, M. A., y Padilla V. (2007). La inteligencia emocional está relacionada con el rendimiento académico. Revista Psicología y Educación, 1 (1), 54-66. 